Research Article

\title{
Enveloping Lie Superalgebras and Killing-Ricci Forms of Bol Superalgebras
}

\author{
Sylvain Attan \\ Département de Mathématiques, Université d'Abomey-Calavi, 01 BP 4521, Cotonou 01, Benin \\ Correspondence should be addressed to Sylvain Attan; syltane2010@yahoo.fr
}

Received 6 August 2020; Accepted 10 March 2021; Published 28 March 2021

Academic Editor: Ljubisa Kocinac

Copyright (C) 2021 Sylvain Attan. This is an open access article distributed under the Creative Commons Attribution License, which permits unrestricted use, distribution, and reproduction in any medium, provided the original work is properly cited.

In this paper, enveloping Lie superalgebras of Bol superalgebras is introduced. The notion of Killing-Ricci forms and invariant forms of these superalgebras is investigated as generalization of the one of Bol algebras.

\section{Introduction}

Bol algebras were introduced [1] in the context of a study of smooth Bol loops. The algebras play the same role with respect to Bol loops as Lie algebras do with respect to Lie groups or Malcev algebras to Moufang loops [2].

A vector space $V$ equipped with a trilinear operation [,,] is called a Lie triple system if $[a, a, b]=0,[a, b, c]+[b$, $c, a]+[c, a, b]=0,[x, y,[a, b, c]]=[[x, y, a], b, c]+[a,[x$, $y, b], c]+[a, b,[x, y, c]]$ for all $x, y, a, b, c \in V$. A (left) Bol algebra $(V, \cdot,[,]$,$) is a Lie triple system (V,[,]$,$) with an$ additional bilinear skew-symmetric operation $\cdot$ satisfying $[a, b, c \cdot d]=[a, b, c] \cdot d+c \cdot[a, b, d]+[c, d, a \cdot b]+(a \cdot b)$ $\cdot(c \cdot d)$. A related notion is that of a Lie triple algebra, introduced under the name generalized Lie triple systems, by Yamaguti [3], and called later as Lie Yamaguti algebras [4].

From the standard enveloping Lie algebra of a given Bol algebra, the notion of Killing-Ricci form and invariant form for a Bol algebra are introduced and studied in [5].

A $\mathbb{Z}_{2}$-graded generalization of Lie algebras, called Lie superalgebras, is considered in $[6,7]$, while a $\mathbb{Z}_{2}$-graded generalization of Lie Yamaguti algebras called Lie Yamaguti superalgebras was first considered in [8] and generalizes Lie supertriple systems [9]. The reader may refer to [10] for applications of Lie supertriple systems in physics. As Lie Yamaguti superalgebras, Bol superalgebras first introduced in [11] may also be viewed as a generalization of Lie supertriple systems. For relations between
Malcev superalgebras and Bol superalgebras, one may refer to [12].

As a part of the general theory of superalgebras, the notion of Killing form of Lie algebras is extended to one of Lie triple systems [13], Lie superalgebras [7], Lie supertriple systems $[14,15]$, and next Lie Yamaguti superalgebras [16].

In this paper, we define enveloping Lie superalgebras and the Killing-Ricci form of Bol superalgebras and study this Killing-Ricci form, which could be seen as a generalization of the one of Bol algebras [5] and the Killing form of Lie supertriple systems $[14,15]$. Unlike Bol algebras, in Bol superalgebras, there is an odd subspace, which is not a Bol subalgebra. This complicates the work more than Bol algebras case.

The rest of this paper is organized as follows. In section 2, we first recall some basics on Lie and Malcev superalgebras as well as Lie supertriple systems and Bol superalgebras. In Section 3, we define the notion of pseudo-superderivations (Definition 8), study their properties (Lemma 1), and introduce the notion of enveloping Lie superalgebras of Bol superalgebras (Definition 12). In Section 4, the Killing-Ricci form of Bol superalgebras is defined (Definition 13) and some of its properties are investigated (Theorem 1, Proposition 3, and Lemma 3). In the next section, the invariant form of Bol superalgebras is defined (Definition 14) and some results are obtained (Lemma 4 and Theorem 2). 
Throughout this paper, all vector superspaces and superalgebras are finite dimensional over a fixed ground field $\mathbb{K}$ of characteristic 0 .

\section{Some Basics on Superalgebras}

We recall here some useful definitions and examples of Lie supertriple systems as well as the one of Bol superalgebras. These examples are obtained from the relation between Malcev superalgebras and Bol superalgebras, which could be found in [12].

Now, let $M$ be a linear superspace over $\mathbb{K}$, that is, a $\mathbb{Z}_{2}$-graded linear space with a direct sum $M=M_{0} \oplus M_{1}$. The elements of $M_{j}, j \in \mathbb{Z}_{2}$, are said to be homogeneous of parity $j$. The parity of a homogeneous element $x$ is denoted by $\bar{x}$. For all $i, j \in \mathbb{Z}_{2}, i+j$ will always means that this sum is calculated modulo 2. If $N=N_{0} \oplus N_{1}$ is another superspace, a linear map $f: M \longrightarrow N$ is said to be of degree $r \in \mathbb{Z}_{2}$ if $f\left(M_{i}\right) \subseteq N_{i+r}$ for all $i \in \mathbb{Z}_{2}$. If $f$ is of degree $r=0$, that is,
$f\left(M_{i}\right) \subseteq N_{i}$ for all $i \in \mathbb{Z}_{2}$, then $f$ is said to be an even linear map. An algebra $(A,[]$,$) is called a superalgebra if the$ underlying vector space is $\mathbb{Z}_{2}$-graded, i.e., $A=A_{0} \oplus A_{1}$ and if furthermore $\left[A_{i}, A_{j}\right] \subset A_{i+j}$. For any binary operation, we will sometimes use juxtaposition in order to reduce the number of braces; i.e., for “.”, $x y \cdot z$ means $(x \cdot y) \cdot z$.

Definition 1. A Lie superalgebra is the superalgebra $\left(A=A_{0} \oplus A_{1},[],\right)$ satisfying the superskew-symmetry and the super-Jacobi identities that is

$$
[x, y]=-(-1)^{\overline{x y}}[y, x]
$$

$[[x, y], z]+(-1)^{\bar{x}(\bar{y}+\bar{z})}[[y, z], x]+(-1)^{\bar{z}(\bar{x}+\bar{y})}[[z, x], y]=0$,

for all $x, y, z \in \mathscr{H}(A)$. In terms of the super-Jacobian,

$$
S J(x, y, z)=[[x, y], z]+(-1)^{\bar{x}(\bar{y}+\bar{z})}[[y, z], x]+(-1)^{\bar{z}(\bar{x}+\bar{y})}[[z, x], y] .
$$

The super-Jacobi identity is written as $S J(x, y, z)=0$ for all $x, y, z \in \mathscr{H}(A)$.

Another class of superalgebras that is of interest in this paper is the one of Malcev superalgebras.
Definition 2 (see $[17,18]$ ). A superalgebra $\left(M=M_{0} \oplus M_{1},[],\right)$ is called a Malcev superalgebra if it satisfies the following superidentities:

$$
\begin{aligned}
{[x, y]=} & -(-1)^{\overline{x y}}[y, x] \text { (superskew- symmetry), } \\
& {[[[x, y], z], t]-[x,[[y, z], t]]-(-1)^{\bar{y}(\bar{z}+\bar{t})}[[x,[z, t]], y]-(-1)^{\bar{t}(\bar{y}+t \bar{z})}[[[x, t], y], z] } \\
= & (-1)^{\overline{y z}}[[x, z],[y, t]] \text { (super - Malcevidentity), }
\end{aligned}
$$

for $x, y, z, t \in \mathscr{H}(M)$.

Definition 3
(1) A supertriple system is a couple $\left(S=S_{0} \oplus S_{1},[,],\right)$ consisting of a $\mathbb{Z}_{2}$-graded $\mathbb{K}$-vector space $S=S_{0} \oplus S_{1}$ and $a \mathbb{K}$-trilinear map ["], satisfying $\left[S_{i}, S_{j}, S_{k}\right] \subset S_{i+j+k}$ for all $i, j, k$ in $\mathbb{Z}_{2}$ such that for all $x, y, z, \in \mathscr{H}(S)$, the following equations hold:

$$
\begin{aligned}
& {[x, y, z]=-(-1)^{\overline{x y}}[y, x, z] \text { (left superskew-symmetry), }} \\
& {[x, y, z]+(-1)^{\bar{x}(\bar{y}+\bar{z})}[y, z, x]+(-1)^{\bar{z}(\bar{x}+\bar{y})}[z, x, y]=0, \text { (superternary - Jacobi identity). }}
\end{aligned}
$$

(2) A Lie supertriple system $[9,19]$ is a supertriple system $\left(S=S_{0} \oplus S_{1},[,],\right)$ such that the superternary Nambu identity

$$
[x, y,[u, v, w]]=[[x, y, u], v, w]+(-1)^{\bar{u}(\bar{x}+\bar{y})}[u,[x, y, v], w]+(-1)^{(\bar{x}+\bar{y})(\bar{u}+\bar{v})}[u, v,[x, y, w]]
$$


holds for all $x, y, u, v, w \in \mathscr{H}(S)$.

Example 1 (see $[9,12,19])$. Let $\left(L=L_{0} \oplus L_{1},[],\right)$ be a Lie superalgebra, then $(L,[,]$,$) is a Lie supertriple system where$ for all $x, y, z \in \mathscr{H}(L),[x, y, z]:=[[x, y], z]$.

Definition 4 (see $[11,12])$. A Bol superalgebra is a triple $(B=$ $\left.B_{0} \oplus B_{1}, \cdot,[,],\right)$ consisting of a superspace $B=B_{0} \oplus B_{1}$, a linear map $: B^{\otimes 2} \longrightarrow B$ satisfying $B_{i} \cdot B_{j} \subseteq B_{i+j}$, and a trilinear map $[,]:, B^{\otimes 3} \longrightarrow B$ satisfying $\left[B_{i}, B_{j}, B_{k}\right] \subseteq B_{i+j+k}, i, j, k \in \mathbb{Z}_{2}$, such that the following holds:

$$
\begin{aligned}
& \left(B S_{1}\right) x \cdot y=-(-1)^{\overline{x y}} y \cdot x . \\
& \left(B S_{2}\right)[x, y, z]=-(-1)^{\overline{x y}}[y, x, z] .
\end{aligned}
$$

$$
\begin{aligned}
& \quad\left(B S_{3}\right)[x, y, z]+(-1)^{\bar{x}(\bar{y}+\bar{z})}[y, z, x]+(-1)^{\bar{z}(\bar{x}+\bar{y})}[z, x, y] \\
& =0 . \\
& \left(B S_{4}\right)[x, y,[u, v, w]]=[[x, y, u], v, w]+(-1)^{\bar{u}(\bar{x}+} \\
& \bar{y})[u,[x, y, v], w]+(-1)^{(\bar{x}+\bar{y})(\bar{u}+\bar{v})}[u, v,[x, y, w]] . \\
& \quad\left(B S_{5}\right)[x, y, u \cdot v]=(-1)^{\bar{u}(\bar{x}+\bar{y})} u \cdot[x, y, v]+[x, y, u] . \\
& \left.v+(-1)^{(\bar{x}+\bar{y})(\bar{u}}+\bar{v}\right)[u, v, x \cdot y]+(x \cdot y) \cdot(u \cdot v) \text { holds for } \\
& \text { all } x, y, u, v, w \in \mathscr{H}(S) .
\end{aligned}
$$

Remark 1. A Bol superalgebra is a Lie triple supersystem $(B=$ $\left.B_{0} \oplus B_{1},[,],\right)$ with a superskew-symmetric binary map. such that $B_{i} \cdot B_{j} \subseteq B_{i+j}$

$$
[x, y, u \cdot v]=(-1)^{\bar{u}(\bar{x}+\bar{y})} u \cdot[x, y, v]+[x, y, u] \cdot v+(-1)^{(\bar{x}+\bar{y})(\bar{u}+\bar{v})}[u, v, x \cdot y]+(x \cdot y) \cdot(u \cdot v)
$$

holds for all $x, y, u, v \in \mathscr{H}(B)$.

$$
[x, y, z]:=\frac{1}{3}\left(2(x \cdot y) \cdot z-(-1)^{\bar{x}(\bar{y}+\bar{z})}(y \cdot z) \cdot x-(-1)^{\bar{z}(\bar{x}+\bar{y})}(z \cdot x) \cdot y\right)
$$

for all $x, y, z \in \mathscr{H}(M)$ becomes a Bol superalgebra ( $\left.M=M_{0} \oplus M_{1}, \cdot,[,],\right)$. This allows us to get the following example of Bol superalgebra from an example of a Malcev superalgebra [20].

Example 2. Let $\left(L^{2}(2,2), \cdot\right)$ be a non-Lie Malcev superalgebra [20] defined with respect to a basis $\left(e_{1}, e_{2}, e_{3}, e_{4}\right)$, where $L^{2}(2,2)_{\overline{0}}=\operatorname{span}\left(e_{1}, e_{2}\right)$ and $L^{2}(2,2)_{\overline{1}}=\operatorname{span}\left(e_{3}, e_{4}\right)$ with the nonzero products given by $e_{1} \cdot e_{2}=e_{2}, e_{1} \cdot e_{3}$ $=e_{3}, e_{1} \cdot e_{4}=-e_{4}$, and $e_{2} \cdot e_{3}=-e_{4}$. Then, $\left(L^{2}(3,1), \cdot,[,],\right)$ is a four-dimensional Bol superalgebra with $L^{2}(2,2)_{\overline{0}}$ $=\operatorname{span}\left(e_{1}, e_{2}\right)$ and $L^{2}(2,2)_{\overline{1}}=\operatorname{span}\left(e_{3}, e_{4}\right)$, where the nonzero products are given by $e_{1} \cdot e_{2}=e_{2}, e_{1} \cdot e_{3}=e_{3}, e_{1} \cdot e_{4}=$ $-e_{4}, e_{2} \cdot e_{3}=-e_{4}$ and $\left[e_{1}, e_{2}, e_{1}\right]=-e_{2},\left[e_{1}, e_{3}, e_{1}\right]=-e_{3}$, $\left[e_{1}, e_{4}, e_{1}\right]=-e_{4}$.

From [12], we also get the following example.

Example 3 (see [12]). There is a four-dimensional Bol superalgebra $\left(L^{2}(3,1), \cdot,[,],\right) \quad$ with $L^{2}(3,1)_{\overline{0}}=\operatorname{span}\left(e_{1}\right.$, $\left.e_{2}, e_{3}\right)$ and $L^{2}(3,1)_{\overline{1}}=\operatorname{span}\left(e_{4}\right)$, where the nonzero products are given by $e_{1} \cdot e_{3}=e_{1}, e_{2} \cdot e_{3}=e_{1}+e_{2}, e_{3} \cdot e_{4}=e_{4}, e_{4} \cdot e_{4}=$ $e_{1}$ and $\left[e_{1}, e_{3}, e_{3}\right]=e_{1},\left[e_{2}, e_{3}, e_{3}\right]=2 e_{1}+e_{2},\left[e_{3}, e_{4}, e_{3}\right]=-e_{4}$.

Definition 5. Let $B=B_{0} \oplus B_{1}$ be a Bol superalgebra. A graded subspace $H=H_{0} \oplus H_{1}$ of $B$ is a subsuperalgebra of $B$ if $H_{i}$. $H_{j} \subseteq H_{i+j}$ and $\left[H_{i}, H_{j}, H_{k}\right] \subseteq H_{i+j+k}$, for all $i, j, k \in \mathbb{Z}_{2}$.
In [12], we proved that any Malcev superalgebra $\left(M=M_{0} \oplus M_{1}\right)$ equipped with a trilinear operation [,] where
Definition 6. A subsuperalgebra $H=H_{0} \oplus H_{1}$ of a Bol superalgebra $B$ is an invariant subsuperalgebra (resp., an ideal) of $B$ if $[B, B, H] \subseteq H$ (resp., $B H \subseteq H$ and $[B, B, H] \subseteq H$ ).

If $H$ is an ideal of $B$, it is an invariant subsuperalgebra of $B$. Obviously, the center of a Bol superalgebra $B$ defined by $Z(B)=\{x \in B, x y=0$ and $[x, y, z]=0, \forall y, z \in B\} \quad$ is an ideal of $B$.

Definition 7. Let $A=A_{0} \oplus A_{1}$ and $B=B_{0} \oplus B_{1}$ be two Bol superalgebras. An even linear map $f: A \longrightarrow B$ is called $a$ morphism of Bol superalgebras if $f(x y)=f(x) f(y)$ and $f([x, y, z])=[f(x), f(y), f(z)]$ for all $x, y, z \in B_{0} \cup B_{1}$.

Recall [6] that if $V=V_{0} \oplus V_{1}$ is a vector superspace, then the set of the linear mappings of $\mathrm{V}$ into itself which are homogeneous of degree $r$ is denoted by $\operatorname{End}_{r}(V)=\left\{f \in \operatorname{End}(V), f\left(V_{i}\right) \subseteq V_{r+i}\right\}$, and we obtain an associative superalgebra End $(V)=\operatorname{End}_{0}(V) \oplus \operatorname{End}_{1}(V)$. The bracket $[f, g]=f g-(-1)^{\bar{f}} \bar{g} g f$ makes $\operatorname{End}(V)$ into a Lie superalgebra which we denote by $l(V)$ or $l(m, n)$ where $m=$ $\operatorname{dim} V_{0}$ and $n=\operatorname{dim} V_{1}$. Let $e_{1}, \ldots, e_{m}, e_{m+1}, \ldots, e_{m+n}$ be a basis of $V$. In this basis, the matrix of $f \in l(m, n)$ is expressed as $\left(\begin{array}{ll}\alpha & \beta \\ \gamma & \delta\end{array}\right), \alpha$ being an $(m \times m)-, \delta$ an $(n \times n)-, \beta$ an $(m \times n)-$, and $\gamma$ an $(n \times m)-$ matrix. The matrices of even elements have the form $\left(\begin{array}{ll}\alpha & 0 \\ 0 & \delta\end{array}\right)$ and those of odd ones $\left(\begin{array}{ll}0 & \beta \\ \gamma & 0\end{array}\right)$. For $f=\left(\begin{array}{ll}\alpha & \beta \\ \gamma & \delta\end{array}\right)$, the supertrace of $M$ is defined by 
$\operatorname{str}(M)=\operatorname{tr} \alpha-\operatorname{tr} \delta$ and does not depend on the choice of a homogeneous basis. We have $\operatorname{str}([f, g])=0$; that is, $\operatorname{str}(f g)=(-1)^{\bar{f} \bar{g}} \operatorname{str}(g f)$ and $\operatorname{str}\left(h f h^{-1}\right)=\operatorname{str}(f)$.

\section{Enveloping Lie Superalgebras of a Bol Superalgebra}

As derivations for algebras, superderivations of different superalgebras are an important subject of study in superalgebras and diverse area. They appear in many fields of mathematics and physics. In particular, they allow the construction of new superalgebras structures. In the case of Bol superalgebras, instead of superderivations, we have the notion of pseudo-superderivations. They generalize pseudoderivations for Bol algebras [2] and superderivations for Lie supertriple systems [19] and allow the construction of enveloping Lie superalgebras of Bol superalgebras.

Definition 8. Let $B=B_{0} \oplus B_{1}$ be a Bol superalgebra. A linear map $P \in \operatorname{End}_{r}(B)$ is called a pseudo-superderivation of companion $a \in B_{r}\left(r \in \mathbb{Z}_{2}\right)$ if, for any $x, y, z \in B_{0} \cup B_{1}$ :

$$
\begin{gathered}
P([x, y, z])=[P(x), y, z]+(-1)^{r \bar{x}}[x, P(y), z]+(-1)^{r(\bar{x}+\bar{y})}[x, y, P(z)], \\
P(x y)=(-1)^{r \bar{x}} x P(y)+P(x) y+(-1)^{r(\bar{x}+\bar{y})}[x, y, a]+a \cdot x y .
\end{gathered}
$$

Remark 2. Note that a pseudo-superderivation $P$ can have more than one companion. Let denote the set of all companions of a pseudo-superderivation $P$ by $\operatorname{Com}(P)$.

Let $p S_{r}(B)$ be the set of all pseudo-superderivations of degree $r$ and $p S(B)=p S_{0}(B) \oplus p S_{1}(B)$. Furthermore, let $P S_{r}(B)=\left\{(P, a), P \in p S_{r}(B), a \in \operatorname{Com}(P)\right\}$ and $P S(B)=P$ $S_{0}(B) \oplus P S_{1}(B)$.

For any pseudo-superderivations $P, Q \in p S_{0}(B) \cup p S_{1}$ $(B)$ of a Bol superalgebra $B$, consider the supercommutator given by $[P, Q]:=P Q-(-1)^{\overline{P Q}} Q P$. Then, we have the following.
Lemma 1. Let $(P, a) \in P S_{r}(B),(Q, b) \in P S_{r}(B),(R, c) \in P$ $S_{s}(B)$, and $\lambda \in \mathbb{K}$. Then, the following holds:

(1) $(P, a)+\lambda(Q, b):=(P+\lambda Q, a+\lambda b) \in P S_{r}(B)$.

(2) $[(P, a),(R, c)]:=\left([P, R], P(c)-(-1)^{r s} R(a)-a c\right) \epsilon$ $P S_{r+s}(B)$.

Proof. Let $\quad(P, a) \in P S_{r}(B), \quad(Q, b) \in P S_{r}(B)$, $(R, c) \in P S_{s}(B)$, and $\lambda \in \mathbb{K}$. The first statement is a straightforward computation. For the second statement, pick $x, y, z, u, v \in B_{0} \cup B_{1}$. Then, using repeatedly 9 for $P$ and $R$, we prove 9 for $[P, R]$ as follows:

$$
\begin{aligned}
& {[P, R]([x, y, w])=P R([x, y, w])-(-1)^{r s} R P([x, y, w])} \\
& =P\left([R(x), y, z]+(-1)^{s \bar{x}}[x, R(y), z]+(-1)^{s(\bar{x}+\bar{y})}[x, y, R(z)]\right) \\
& -(-1)^{r s} R\left([P(x), y, z]+(-1)^{r \bar{x}}[x, P(y), z]+(-1)^{r(\bar{x}+\bar{y})}[x, y, P(z)]\right) \\
& =[P R(x), y, z]+(-1)^{r(\bar{x}+s)}[R(x), P(y), z]+(-1)^{r(s+\bar{x}+\bar{y})}[R(x), y, P(z)] \\
& +(-1)^{s \bar{x}}[P(x), R(y), z]+(-1)^{(r+s) \bar{x}}[x, P R(y), z] \\
& \left.+(-1)^{s \bar{x}+r(\bar{x}+\bar{y}+s)}[x, R(y), P(z)]+(-1)^{s(\bar{x}+\bar{y})}[P(x), y, R(z)]\right) \\
& \left.\left.+(-1)^{s(\bar{x}+\bar{y})+r \bar{x}}[x, P(y), R(z)]\right)+(-1)^{(r+s)(\bar{x}+\bar{y}))}[x, y, P R(z)]\right) \\
& -(-1)^{r s}\{[R P(x), y, z]+(-1))^{s(\bar{x}+r)}[P(x), R(y), z] \\
& +(-1)^{s(r+\bar{x}+\bar{y})}[P(x), y, R(z)]+(-1)^{r \bar{x}}[R(x), P(y), z]+(-1)^{(r+s) \bar{x}}[x, R P(y), z] \\
& \left.+(-1)^{r \bar{x}+s(\bar{x}+\bar{y}+r)}[x, P(y), R(z)]+(-1)^{r(\bar{x}+\bar{y})}[R(x), y, P(z)]\right) \\
& \left.\left.\left.+(-1)^{r(\bar{x}+\bar{y})+s \bar{x}}[x, R(y), P(z)]\right)+(-1)^{(r+s)(\bar{x}+\bar{y}))}[x, y, R P(z)]\right)\right\} \\
& =[[P, R](x), y, z]+(-1)^{(r+s) \bar{x}}[x,[P, R](y), z]+(-1)^{(r+s)(\bar{x}+\bar{y})}[x, y,[P, R](z)] \text {. }
\end{aligned}
$$

Thus, we get (9) for $[P, R]$. To prove, $(10)$ for $[P, R]$, note that if we use repeatedly (10) for $P$, we get 


$$
\begin{aligned}
P(c \cdot x y)= & (-1)^{r s} c \cdot P(x y)+P(c) \cdot x y+(-1)^{r(\bar{x}+\bar{y}+s}[c, x y, a]+a \cdot(c \cdot x y) \\
= & +(-1)^{r(\bar{x}+s)} c \cdot x P(y)+(-1)^{r s} c \cdot P(x) y+(-1)^{r(\bar{x}+\bar{y}+s)} c \cdot[x, y, a] \\
& +(-1)^{r s} c \cdot(a \cdot x y)+P(c) \cdot x y+(-1)^{r(\bar{x}+\bar{y}+s)}[c, x y, a]+a \cdot(c \cdot x y),
\end{aligned}
$$

and therefore, by (9) and (12) for $P$ and $R$, we get

$$
\begin{aligned}
& {[P, R](x y)=P R(x y)-(-1)^{r s} R P(x y)} \\
& =P\left((-1)^{s \bar{x}} x R(y)+R(x) y+(-1)^{s(\bar{x}+\bar{y})}[x, y, c]+c \cdot x y\right) \\
& -(-1)^{r s}\left\{R\left((-1)^{r \bar{x}} x P(y)+P(x) y+(-1)^{r(\bar{x}+\bar{y})}[x, y, a]+a \cdot x y\right)\right\} \\
& =(-1)^{(r+s) \bar{x}} x P R(y)+(-1)^{s \bar{x}} P(x) R(y)+(-1)^{r(\bar{x}+\bar{y}+s)+s \bar{x}}[x, R(y), a] \\
& +(-1)^{s \bar{x}} a \cdot x R(y)+(-1)^{r(\bar{x}+s)} R(x) P y+P R(x) P(y)+(-1)^{r(\bar{x}+\bar{y}+s)}[R(x), y, a] \\
& a \cdot R(x) y+(-1)^{s(\bar{x}+\bar{y})}[P(x), y, c]+(-1)^{s(\bar{x}+\bar{y})+r \bar{x}}[x, P(y), c] \\
& +(-1)^{(r+s)(\bar{x}+\bar{y})}[x, y, P(c)]+(-1)^{r(\bar{x}+s)} c \cdot x P(y)+(-1)^{r s} c \cdot P(x) y \\
& +(-1)^{r(\bar{x}+\bar{y}+s)} c \cdot[x, y, a]+(-1)^{r s} c \cdot(a \cdot x y)+P(c) \cdot x y+(-1)^{r(\bar{x}+\bar{y}+s)}[c, x y, a] \\
& +a \cdot(c \cdot x y)-(-1)^{r s}\left\{(-1)^{(r+s) \bar{x}} x R P(y)+(-1)^{r \bar{x}} R(x) P(y)\right. \\
& +(-1)^{s(\bar{x}+\bar{y}+r)+r \bar{x}}[x, P(y), c]+(-1)^{r \bar{x}} c \cdot x P(y)+(-1)^{s(\bar{x}+r)} P(x) R y+R P(x)(y) \\
& +(-1)^{s(\bar{x}+\bar{y}+r)}[P(x), y, c]+c \cdot P(x) y+(-1)^{r(\bar{x}+\bar{y})}[R(x), y, a] \\
& +(-1)^{r(\bar{x}+\bar{y})+s \bar{x}}[x, R(y), a]+(-1)^{(r+s)(\bar{x}+\bar{y})}[x, y, R(a)]+(-1)^{s(\bar{x}+r)} a \cdot x R(y) \\
& +(-1)^{r s} a \cdot R(x) y+(-1)^{s(\bar{x}+\bar{y}+r)} a \cdot[x, y, c]+(-1)^{r s} a \cdot(c \cdot x y)+R(a) \cdot x y \\
& \left((-1)^{s(\bar{x}+\bar{y}+r)}[a, x y, c]+c \cdot(a \cdot x y)\right\} \\
& =(-1)^{(r+s) \bar{x}} x \cdot[P, R](y)+[P, R](x) \cdot y+(-1)^{(r+s)(\bar{x}+\bar{y})}\left[x, y, P(c)-(-1)^{r s} R(a)\right] \\
& +\left(P(c)-(-1)^{r s} R(a)\right) \cdot x y+(-1)^{r(\bar{x}+\bar{y}+s)}[c, x y, a]-(-1)^{s(\bar{x}+\bar{y})}[a, x y, c] \\
& +(-1)^{r(\bar{x}+\bar{y}+s)} c[x, y, a]-(-1)^{s(\bar{x}+\bar{y})} a[x, y, c] \\
& =(-1)^{(r+s) \bar{x}} x \cdot[P, R](y)+[P, R](x) \cdot y+(-1)^{(r+s)(\bar{x}+\bar{y})}\left[x, y, P(c)-(-1)^{r s} R(a)\right] \\
& +\left(P(c)-(-1)^{r s} R(a)\right) \cdot x y-(-1)^{(r+s)(\bar{x}+\bar{y})+r s}[x y, c, a]-(-1)^{s(\bar{x}+\bar{y})}[a, x y, c] \\
& -(-1)^{(r+s)(\bar{x}+\bar{y})}[x, y, a] c-(-1)^{s(\bar{x}+\bar{y})} a[x, y, c]\left(\text { by }\left(B S_{1}\right) \text { and }\left(B S_{2}\right)\right) \\
& =(-1)^{(r+s) \bar{x}} x \cdot[P, R](y)+[P, R](x) \cdot y+(-1)^{(r+s)(\bar{x}+\bar{y})}\left[x, y, P(c)-(-1)^{r s} R(a)-a c\right] \\
& +\left(P(c)-(-1)^{r s} R(a)-a c\right) \cdot x y\left(\text { by }\left(B S_{1}\right)-\left(B S_{3}\right) \text { and }\left(B S_{5}\right)\right) \text {, }
\end{aligned}
$$

and then, we get (10). This ends the proof.

Now, we can prove the following result.

Proposition 1. Let $B=B_{0} \oplus B_{1}$ be a Bol superalgebra. Then, $(P S(B),[]$,$) is a Lie superalgebra.$

Proof. The proof follows by Lemma 1 and the fact that $p S(B)$ is a Lie superalgebra since it is straightforward to check that $p S(B)$ is a subsuperalgebra of the Lie superalgebra End $(B)$.

Definition 9. PS $(B)$ is called the enlarged Lie superalgebra of pseudo-superderivations of a Bol superalgebra $B$.

Let $B=B_{0} \oplus B_{1}$ be a Bol superalgebra. For any $x, y \in B_{0} \cup B_{1}$, denote by $D_{x, y}$, the endomorphism of $B$ defined by $D_{x, y}(z):=[x, y, z]$ for all $z \in B$. We have, for any 
$x, y \in B_{0} \cup B_{1}, r \in \mathbb{Z}_{2}, D_{x, y}\left(B_{r}\right) \in B_{r+\bar{x}+\bar{y}}$; that is, $D_{x, y}$ is a linear map of degree $\bar{x}+\bar{y}$. Moreover, it comes from $\left(B S_{4}\right)$ and $\left(B S_{5}\right)$ that

$$
\begin{aligned}
D_{x, y}([u, v, w]) & =\left[D_{x, y}(u), v, w\right]+(-1)^{\bar{u}(\bar{x}+\bar{y})}\left[u, D_{x, y}(v), w\right]+(-1)^{(\bar{x}+\bar{y})(\bar{u}+\bar{v})}\left[u, v, D_{x, y}(w)\right] \\
D_{x, y}(u \cdot v) & =(-1)^{\bar{u}(\bar{x}+\bar{y})} u \cdot D_{x, y}(v)+D_{x, y}(u) \cdot v+(-1)^{(\bar{x}+\bar{y})(\bar{u}+\bar{v})}[u, v, x \cdot y]+(x \cdot y) \cdot(u \cdot v),
\end{aligned}
$$

for any $x, y, u, v$ in $B_{0} \cup B_{1}$. It follows that $D_{x, y}$ is pseudosuperderivation of degree $\bar{x}+\bar{y}$ and companion $x y$, called inner pseudo-superderivation of $B$.

Now, one can reformulate the definition of a Bol superalgebra in the following manner.

Definition 10. A vector superspace $B=B_{0} \oplus B_{1}$ equipped with a bilinear operation $(x, y) \mapsto x \cdot y$ satisfying $B_{i} \cdot B_{j} \subseteq B_{i+j}$ and a trilinear operation $(x, y, z) \mapsto[x, y, z]$ satisfying $\left[B_{i}, B_{j}, B_{k}\right] \subseteq B_{i+j+k} ; i, j, k \in \mathbb{Z}_{2}$, is called a Bol superalgebra if the following holds:

$\left(B S_{1}\right) x \cdot y=-(-1)^{\overline{x y}} y \cdot x$.

$\left(B S_{2}\right)[x, y, z]=-(-1)^{\overline{x y}}[y, x, z]$.

$\left(B S_{3}\right)[x, y, z]+(-1)^{\bar{x}(\bar{y}+\bar{z})}[y, z, x]+(-1)^{\bar{z}(\bar{x}+\bar{y})}[z, x$, $y]=0$, and the endomorphism $D_{x, y}: z \mapsto[x, y, z]$ is its pseudo-superderivation with a companion $x y$ for all $x, y \in B_{0} \cup B_{1}$ and $z \in B_{0} \cup B_{1}$.

Let $i p S_{r}(B, B)$ be the vector space spanned by all inner pseudo-superderivations

$D_{x, y}\left(x, y \in B_{0} \cup B_{1}\right.$ and $\left.\bar{x}+\bar{y}=r \in \mathbb{Z}_{2}\right)$.

We can define naturally a $\mathbb{Z}_{2}$-gradation by setting $i p S(B, B)=i p S_{0}(B, B) \oplus i p S_{1}(B, B)$. Evidently, $i p S(B, B)$ is a subsuperalgebra of the Lie superalgebra $p S(B)$. Accordingly, $\operatorname{IPS}(B, B)$ can be introduced as the set of all pairs $(P, c)$, where $P \in i p S(B, B)$ and $c \in \operatorname{Com}(P)$. Evidently, $\operatorname{IPS}(B, B)$ is a subsuperalgebra of the Lie superalgebra $P S(B)$.

Definition 11. IPS $(B, B)$ is called the enlarged Lie superalgebra of inner pseudo-superderivations.

Let $B=B_{0} \oplus B_{1}$ be a Bol superalgebra and $H=H_{0} \oplus H_{1}$ be a subsuperalgebra of $P S(B)$ such that $\operatorname{IPS}(B, B) \subseteq H$. For $i \in \mathbb{Z}_{2}$, let $\mathscr{L}_{i}(B)=B_{i} \oplus H_{i}$ and define a new superbracket operation in $\mathscr{L}^{H}(B)=\mathscr{L}_{0}(B) \oplus \mathscr{L}_{1}(B)=B \oplus H$ as follows: for any $x, y \in B_{0} \cup B_{1},(P, a),(Q, b) \in H_{0} \cup H_{1}$ :

$$
\begin{aligned}
{[x, y] } & :=\left(D_{x, y}, x y\right), \\
{[(P, a), x] } & :=-(-1)^{\overline{a x}}[x,(P, a)]:=P(x), \\
{[(P, a),(Q, b)] } & :=\left([P, Q], P(b)-(-1)^{\bar{a} \bar{b}} Q(a)-a b\right) .
\end{aligned}
$$

Then, we have the following.

Proposition 2. $\left(\mathscr{L}^{H}(B),[],\right)$ is a Lie superalgebra.

Proof. It is clear that the operation [,] is supersymmetric. For the Jacobi superidentity, there are many cases to distinguish. First, for all $x, y, u$ in $B_{0} \cup B_{1},(P, a),(Q, b)$ in $H_{0} \cup H_{1}$, we get

$$
\begin{aligned}
& \left([[x, y],(P, a)]+(-1)^{\bar{x}(\bar{y}+\bar{a})}[[y,(P, a)], x]+(-1)^{\bar{a}(\bar{x}+\bar{y})}[[(P, a), x], y]\right)(u) \\
& =[x, y, P(u)]-(-1)^{\bar{a}(\bar{x}+\bar{y})} P([x, y, u])-(-1)^{\bar{x}(\bar{a}+\bar{y})+\bar{a} \bar{y}}[P(y), x, u]+(-1)^{\bar{a}(\bar{x}+\bar{y})}[P(x), y, u] \\
& =-(-1)^{\bar{a}(\bar{x}+\bar{y})}\left(-(-1)^{\bar{a}(\bar{x}+\bar{y})}[x, y, P(u)]+P([x, y, u])-(-1)^{\overline{a x}}[x, P(y), u]-[P(x), y, u]\right) \\
& =0(\operatorname{by}(9)),
\end{aligned}
$$

i.e., $[[x, y],(P, a)]+(-1)^{\bar{x}(\bar{y}+\bar{a})}[[y,(P, a)], x]+(-1)^{\bar{a}(\bar{x}+\bar{y})}$

$[[(P, a), x], y]=0$.

$$
\begin{aligned}
& {[[(P, a), x],(Q, b)]+(-1)^{\bar{a}(\bar{x}+\bar{b})}[[x,(Q, b)],(P, a)]+(-1)^{\bar{b}(\bar{a}+\bar{x})}[[(Q, b),(P, a)], x]} \\
& =P Q(x)-(-1)^{\bar{a} \bar{b}} Q P(x)+(-1)^{\bar{a}(\bar{x}+\bar{b})}[Q(x),(P, a)]-(-1)^{\bar{x} \bar{b}}[P(x),(Q, b)] \\
& =P Q(x)-(-1)^{\bar{a} \bar{b}} Q P(x)-P Q(x)+(-1)^{\bar{a} \bar{b}} Q P(x)=0 .
\end{aligned}
$$


Next, the other cases when the three elements are in $B_{0} \cup B_{1}$ or $H_{0} \cup H_{1}$ follow from $\left(H B S_{3}\right)$ and the fact that $H$ is a Lie superalgebra.

Definition 12. An enveloping superalgebra of a Bol superalgebra $B$ is a Lie superalgebra $\mathscr{L}^{H}(B)$ defined above. Taking $H=P S(B)$, we obtain the maximal enveloping superalgebra, and taking $H=\operatorname{IPS}(B, B)$, we obtain the minimal (standard) enveloping superalgebra.

The following result will be used in the last section.

Lemma 2. Let $K$ be an ideal of a Bol superalgebra $B$. Then, $\mathscr{K}=K \oplus I P S(B, K)$ is an ideal of the standard enveloping superalgebra $L=B \oplus I P S(B, B)$.

Proof. It suffices to prove that $[\mathscr{K}, L] \subseteq \mathscr{K}$, which is a straightforward computation.

\section{Killing-Ricci Forms of Bol Superalgebras}

The definition of the Killing-Ricci form given here for Bol superalgebras stems from [5], where the Killing-Ricci form for Bol algebras is defined following [21] as the restriction of the Killing form of the standard enveloping Lie algebra of the given Bol algebra to this later. Let $B=B_{0} \oplus B_{1}$ be an $n$-dimensional Bol superalgebra and $L(B)=\left(B_{0} \oplus I P S_{0}(B, B)\right)$ $\oplus\left(B_{1} \oplus I P S_{1}(B, B)\right):=B \oplus I P S(B, B)$ its standard enveloping Lie superalgebra. Let $\alpha$ be the Killing form of $L(B)$ and $\beta$, the restriction of $\alpha$ to $B \times B$. From [5], we introduce the following definition.

Definition 13. The form $\beta$ is called the Killing-Ricci form of the Bol superalgebra $B$.
For any $x, y \in B_{0} \cup B_{1}$, define the endomorphism $R_{x, y}$ of the vector superspace $B$ by $R_{x, y}(z)=(-1)^{\bar{z}(\bar{x}+\bar{y})}[z, x, y]=$ $(-1)^{\bar{z}(\bar{x}+\bar{y})} D_{z, x}(y)$ for all $z \in B_{0} \cup B_{1}$. It is clear that $R_{x, y}$ is of degree $\bar{x}+\bar{y}$.

The next result gives an explicit expression of $\beta$.

Theorem 1. For every $x, y \in B_{0} \cup B_{1}$,

$$
\beta(x, y)=\operatorname{str}\left(R_{x, y}+(-1)^{\overline{x y}} R_{y, x}\right) .
$$

Proof. Let $\left\{e_{i}\right\},\left\{f_{i}\right\},\left\{u_{i}\right\}$, and $\left\{v_{i}\right\}$ be bases for $B_{0}, B_{1}$, $I_{P S_{0}}(B, B)$, and $I P S_{1}(B, B)$, respectively. It suffices to prove (20) for all elements $x, y$ in the basis, i.e., $\beta\left(e_{i}, e_{j}\right)=\alpha\left(e_{i}, e_{j}\right)$, $\beta\left(e_{i}, f_{j}\right)=\alpha\left(e_{i}, f_{j}\right)$, and $\beta\left(f_{i}, f_{j}\right)=\alpha\left(f_{i}, f_{j}\right)$. For these bases, we express the operations of $B$ and $\operatorname{ISP}(B, B)$, using the tensor notation (i.e., repeated indices imply summation), as follows:

$$
\begin{aligned}
D_{e_{i}, e_{j}} & =R_{i j}^{m} u_{m}, \\
D_{e_{i}, f_{j}} & =S_{i j}^{m} v_{m}, \\
D_{f_{i}, f_{j}} & =T_{i j}^{m} u_{m}, \\
{\left[u_{m}, e_{i}\right] } & =u_{m}\left(e_{i}\right)=C_{m i}^{j} e_{j}, \\
{\left[v_{m}, e_{i}\right] } & =v_{m}\left(e_{i}\right)=H_{m i}^{j} f_{j}, \\
{\left[u_{m}, f_{i}\right] } & =u_{m}\left(f_{i}\right)=K_{m i}^{j} f_{j}, \\
{\left[v_{m}, f_{i}\right] } & =v_{m}\left(f_{i}\right)=L_{m i}^{j} e_{j} .
\end{aligned}
$$

Since $R_{e_{i}, f_{j}}\left(B_{0}\right) \subseteq B_{1}$ and $R_{e_{i}, f_{j}}\left(B_{1}\right) \subseteq B_{0}$, we have $\operatorname{str}\left(R_{e_{i}, f_{j}}+R_{f_{j}, e_{i}}\right)=0$ and then $\beta\left(e_{i}, f_{j}\right)=0=\alpha\left(e_{i}, f_{j}\right)$ (consistency property of $\alpha$ ). Hence, it remains to show that $\beta\left(e_{i}, e_{j}\right)=\alpha\left(e_{i}, e_{j}\right)$ and $\beta\left(f_{i}, f_{j}\right)=\alpha\left(f_{i}, f_{j}\right)$. The identities (15) and (16) imply the following:

$$
\begin{aligned}
& L_{e_{i}} L_{e_{j}}\left(e_{k}\right)=\left[e_{i},\left[e_{j}, e_{k}\right]\right]=\left[e_{i}, D_{e_{j}, e_{k}}\right]=D_{e_{k}, e_{j}}\left(e_{i}\right)=R_{k j}^{m} C_{m i}^{t} e_{t}, \\
& L_{e_{i}} L_{e_{j}}\left(f_{k}\right)=\left[e_{i},\left[e_{j}, f_{k}\right]\right]=\left[e_{i}, D_{e_{j}, f_{k}}\right]=-D_{e_{j}, f_{k}}\left(e_{i}\right)=-S_{j k}^{m} H_{m i}^{t} f_{t}, \\
& L_{e_{i}} L_{e_{j}}\left(u_{m}\right)=\left[e_{i},\left[e_{j}, u_{m}\right]\right]=-\left[e_{i}, u_{m}\left(e_{j}\right)\right]=-C_{m j}^{k}\left[e_{i}, e_{k}\right]=-C_{m j}^{k} R_{i k}^{t} u_{t}, \\
& L_{e_{i}} L_{e_{j}}\left(v_{m}\right)=\left[e_{i},\left[e_{j}, v_{m}\right]\right]=-\left[e_{i}, v_{m}\left(e_{j}\right)\right]=-H_{m j}^{k}\left[e_{i}, e_{k}\right]=-H_{m j}^{k} S_{i k}^{t} v_{t} . \\
& \beta\left(e_{i}, e_{j}\right)=R_{k j}^{m} C_{m i}^{k}+S_{j k}^{m} H_{m i}^{k}-C_{m j}^{k} R_{i k}^{m}+H_{m j}^{k} S_{i k}^{m} .
\end{aligned}
$$
we get

Thus, from the relation $\beta\left(e_{i}, e_{j}\right)=\alpha\left(e_{i}, e_{j}\right)=\operatorname{str}\left(L_{e_{i}} L_{e_{j}}\right)$,

From the other hand, we have

$$
\begin{aligned}
& R_{e_{i}, e_{j}}\left(e_{k}\right)=D_{e_{k}, e_{i}}\left(e_{j}\right)=-D_{e_{i}, e_{k}}\left(e_{j}\right)=-R_{i k}^{m} u_{m}\left(e_{j}\right)=-R_{i k}^{m} C_{m j}^{t} e_{t}, \\
& R_{e_{i}, e_{j}}\left(f_{k}\right)=D_{f_{k}, e_{i}}\left(e_{j}\right)=-D_{e_{i}, f_{k}}\left(e_{j}\right)=-S_{i k}^{m} v_{m}\left(e_{j}\right)=-S_{i k}^{m} H_{m j}^{t} f_{t} .
\end{aligned}
$$

By interchanging $i$ and $j$, we have 


$$
R_{e_{j}, e_{i}}\left(e_{k}\right)=-R_{j k}^{m} C_{m i}^{t} e_{t}=R_{k j}^{m} C_{m i}^{t} e_{t}, \quad \text { and } R_{e_{j}, e_{i}}\left(f_{k}\right)=-R_{j k}^{m} H_{m i}^{t} f_{t} \text {. }
$$

Then, we get

$$
\operatorname{str}\left(R_{e_{i}, e_{j}}+(-1)^{\overline{e i} \bar{e}_{j}} R_{e_{j} e_{i}}\right)=-R_{i k}^{m} C_{m j}^{k}+S_{i k}^{m} H_{m j}^{k}+R_{k j}^{m} C_{m i}^{k}+R_{j k}^{m} H_{m i}^{k} .
$$

From (23) and (26), we obtain

Again, the identities (15) and (16) imply the following:

$$
\beta\left(e_{i}, e_{j}\right)=\operatorname{str}\left(R_{e_{i}, e_{j}}+(-1)^{\bar{e} i \overline{e_{j}}} R_{e_{j}, e_{i}}\right) .
$$

$$
\begin{aligned}
L_{f_{i}} L_{f_{j}}\left(e_{k}\right) & =\left[f_{i},\left[f_{j}, e_{k}\right]\right]=-\left[f_{i}, D_{e_{k}, f_{j}}\right]=-D_{e_{k}, f_{j}}\left(f_{i}\right)=-S_{k j}^{m} v_{m}\left(f_{i}\right) \\
& =-S_{k j}^{m} L_{m i}^{t} e_{t}, \\
L_{f_{i}} L_{f_{j}}\left(f_{k}\right)= & {\left[f_{i},\left[f_{j}, f_{k}\right]\right]=\left[f_{i}, D_{f_{j}, f_{k}}\right]=-D_{f_{j}, f_{k}}\left(f_{i}\right)=-T_{j k}^{m} u_{m}\left(f_{i}\right) } \\
& =-T_{j k}^{m} K_{m i}^{t} f_{t}, \\
L_{f_{i}} L_{f_{j}}\left(u_{k}\right)= & {\left[f_{i},\left[f_{j}, u_{k}\right]\right]=-\left[f_{i}, u_{k}\left(f_{j}\right)\right]=-K_{k j}^{m}\left[f_{i}, f_{m}\right]=-K_{k j}^{m} D_{f_{i}, f_{m}} } \\
& =-K_{k j}^{m} T_{i m}^{s} u_{s}, \\
L_{f_{i}} L_{f_{j}}\left(v_{k}\right) & =\left[f_{i},\left[f_{j}, v_{k}\right]\right]=\left[f_{i}, v_{k}\left(f_{j}\right)\right]=L_{k j}^{m}\left[f_{i}, e_{m}\right]=-L_{k j}^{m} D_{e_{m}, f_{i}} \\
& =-L_{k j}^{m} S_{m i}^{s} v_{s} .
\end{aligned}
$$

Again, from the relation $\beta\left(f_{i}, f_{j}\right)=\alpha\left(f_{i}, f_{j}\right)=\operatorname{str}\left(L_{f_{i}}\right.$ $L_{f_{j}}$, we get

$$
\beta\left(f_{i}, f_{j}\right)=-S_{k j}^{m} L_{m i}^{k}+T_{j k}^{m} K_{m i}^{k}-K_{k j}^{m} T_{i m}^{k}+L_{k j}^{m} S_{m i}^{k} .
$$

From the other hand, we get

$$
\begin{aligned}
& R_{f_{i}, f_{j}}\left(e_{k}\right)=D_{e_{k}, f_{i}}\left(f_{j}\right)=S_{k i}^{m} v_{m}\left(f_{j}\right)=S_{k i}^{m} L_{m j}^{s} e_{s}, \quad \text { and, } \\
& R_{f_{i}, f_{j}}\left(f_{k}\right)=D_{f_{k}, f_{i}}\left(f_{j}\right)=T_{k i}^{m} u_{m}\left(f_{j}\right)=T_{k i}^{m} K_{m j}^{s} f_{s} .
\end{aligned}
$$

By interchanging $i$ and $j$, we get

$$
R_{f_{j}, f_{i}}\left(e_{k}\right)=S_{k j}^{m} L_{m i}^{s} e_{s}, \quad \text { and } R_{f_{j}, f_{i}}\left(f_{k}\right)=T_{k j}^{m} K_{m i}^{s} f_{s}=T_{j k}^{m} K_{m i}^{s} f_{s} .
$$

Next, we get

$$
\begin{aligned}
& \operatorname{str}\left(R_{f_{i}, f_{j}}+(-1)^{\overline{f i f_{j}}} R_{f_{j}, f_{i}}\right)=\operatorname{str}\left(R_{f_{i}, f_{j}}-R_{f_{j}, f_{i}}\right), \\
& =S_{k i}^{m} L_{m j}^{k}-T_{k i}^{m} K_{m j}^{k}-S_{k j}^{m} L_{m i}^{k}+T_{j k}^{m} K_{m i}^{k} \\
& =S_{m i}^{k} L_{k j}^{m}-T_{m i}^{k} K_{k j}^{m}-S_{k j}^{m} L_{m i}^{k}+T_{j k}^{m} K_{m i}^{k},
\end{aligned}
$$

and therefore from (29) and (32), we obtain

$$
\beta\left(f_{i}, f_{j}\right)=\operatorname{str}\left(R_{f_{i}, f_{j}}+(-1)^{\overline{f i f_{j}}} R_{f_{j}, f_{i}}\right),
$$

where by relation (20) is proved.

Remark 3. Recall that if $(B, \cdot,[,]$,$) is a (left) Bol algebra, then$ the Killing-Ricci form on $B$ is defined as $\beta(x, y)=\operatorname{tr}\left(R_{x, y}+\right.$ $R_{y, x}$ ) which we deduce from the one for (right) Bol algebra [5] where $R_{x, y}(z)=[z, x, y]$. So, if a Bol superalgebra $B$ is reduced to a Bol algebra, $\beta$ as in Theorem 1 is the Killing-Ricci form of the Bol algebra $B$.

Proposition 3. Let $B=B_{0} \oplus B_{1}$ be a Bol superalgebra with a Killing-Ricci form $\beta$. Then, the following holds:

(1) $\beta(x, y)=(-1)^{\overline{x y}} \beta(y, x)$ for all $x, y \in B_{0} \cup B_{1}$ (supersymmetry).

(2) $\beta\left(B_{0}, B_{1}\right)=0$ (consistence).

(3) $\beta(A(x), A(y))=\beta(x, y), A \in \operatorname{Aut}(B)$.

(4) $\beta([x, y, z], u)=-(-1)^{\bar{z}(\bar{x}+\bar{y})} \beta(z,[x, y, u])$, for all $x, y, z, u \in B_{0} \cup B_{1}$.

Proof. We know [6] that if $\alpha$ is a Killing form of any Lie superalgebra, then $\alpha$ satisfies the relations (1) - (3) of the proposition above. Then, the relations (1) - (3) follow from the fact that the Killing-Ricci form $\beta$ is the restriction to $B$ of 
the Killing form of the standard enveloping superalgebra $L(B)$ of $B$. For the relation (4), pick $x, y, z, u \in B_{0} \cup B_{1}$ and denote by $\alpha$ the Killing form of $L(B)$. Since $L(B)$ is a Lie superalgebra, then $\alpha$ satisfies

$$
\alpha([x, y], z)=-(-1)^{\overline{x y}} \alpha(y,[x, z]) .
$$

Then,

$$
\begin{aligned}
\beta([x, y, z], u) & =\alpha([[x, y], z], u)=-(-1)^{\bar{z}(\bar{x}+\bar{y})} \\
\alpha(z,[[x, y], u]) & =-(-1)^{\bar{z}(\bar{x}+\bar{y})} \beta(z,[x, y, u]) .
\end{aligned}
$$

Hence, the proposition is proved.

Remark 4. If we consider the Bol superalgebra $B$ as a Lie supertriple system with the ternary operation ["], that we denote by $B_{S}$, then the relation (4) in Proposition 3 says that the Killing-Ricci form of $B$ is an invariance form of $B_{S}$.

The following result will be used below.

Lemma 3. Let $K$ be a Killing-Ricci form of a Bol superalgebra $B$. Then, the following conditions are equivalent:

$$
\begin{aligned}
& K([x, y, z], u)=-(-1)^{\bar{z}(\bar{x}+\bar{y})} K(z,[x, y, u]), \\
& K([x, y, z], u)=-(-1)^{\bar{y}(\bar{z}+\bar{u})} K(x,[z, u, y]), \\
& K(x,[y, z, u])=(-1)^{\overline{x y}+\bar{z} \bar{u}} K(y,[x, u, z]),
\end{aligned}
$$

for all $x, y, z, u \in B_{0} \cup B_{1}$.

Proof. We know that, in any Lie supertriple system with an invariant form, the relations (39)-(41) are inequivalent [14]. The proof then follows from the fact that the Killing-Ricci form of $B$ is an invariant form of $B_{S}$.

\section{Invariant Forms of Bol Superalgebras}

In this section, we introduce the concept of invariant forms of Bol superalgebras as generalization of those of Bol algebras and Lie supertriple systems.

Definition 14. An invariant form $b$ of a Bol superalgebra $B=$ $B_{0} \oplus B_{1}$ is a supersymmetric bilinear form on $B$ satisfying the identities

$$
\begin{gathered}
b(x y, z)=-(-1)^{\overline{x y}} b(y, x z), \\
b([x, y, z], u)=-(-1)^{\bar{y}(\bar{z}+\bar{u})} b(x,[z, u, y]),
\end{gathered}
$$

for all $x, y, z, u \in B_{0} \cup B_{1}$.

Remark 5. If B is reduced to a Lie supertriple system (resp., a (left) Bol algebra), then $b$ is reduced to an invariant form to $a$ Lie supertriple system $[14,15]$, (resp., a (left) Bol algebra) which can be deduced from one of the (right) Bol algebras [5].
Let $b$ be an invariant form of a Bol superalgebra $B=B_{0} \oplus B_{1}$. Then, $b$ is an invariant form of $B_{S}$, and by Lemma $3, b$ satisfies the following equivalent conditions:

$$
\begin{aligned}
& b([x, y, z], u)=-(-1)^{\bar{z}(\bar{x}+\bar{y})} b(z,[x, y, u]), \\
& b([x, y, z], u)=-(-1)^{\bar{y}(\bar{z}+\bar{u})} b(x,[z, u, y]), \\
& b(x,[y, z, u])=(-1)^{\overline{x y}+\bar{z} \bar{u}} b(y,[x, u, z]),
\end{aligned}
$$

for all $x, y, z, u \in B_{0} \cup B_{1}$.

Definition 15. Let $b$ be an invariant form of a Bol superalgebra $B$ and $V$ be a subset of $B$. The orthogonal $V^{\perp}$ of $V$ with respect to $b$ is defined by $V^{\perp}=\{x \in B, b(x, y)=0, \forall y \in V\}$. The invariant form $b$ is nondegenerate if $B^{\perp}=\{0\}$.

Lemma 4. Let b be an invariant form of a Bol superalgebra $B$. Then, the following holds:

(1) $(B+[B, B, B])^{\perp}=Z(B)$ if $b$ is nondegenerate.

(2) If $I$ is an ideal of $B$ then $I^{\perp}$ is an ideal of $B$. In particular, $B^{\perp}$ is an ideal of $B$.

Proof. Pick a homogeneous element $u$ in $(B+[B, B, B])^{\perp}$. Then, for any $x, y, z$ in $B_{0} \cup B_{1}$, we get $b(u, x y)=0$ and $b(u,[x, y, z])=0$. This implies by (37) and (38) that $(-1)^{\overline{u x}} b(x u, y)=0$ and $(-1)^{\bar{z}(\bar{x}+\bar{y})} b([u, z, x], y)=0$. As $b$ is nondegenerate, we obtain $x u=0$ and $[u, z, x]=0$ for any $x, z$ in $B_{0} \cup B_{1}$, and this gives $u \in Z(B)$.

Conversely, if $u \in Z(B)$, we have for all $x, y, z, v, w$ in $B$, $b(u, x y+[z, v, w])=b(u, x y)+b(u,[z, v, w])=0$ and $u \in$ $(B+[B, B, B])^{\perp}$ whence $(B+[B, B, B])^{\perp}=Z(B)$.

Now, suppose that $I$ is an ideal of $B$ that is $B I \subseteq I$, $[B, I, B] \subseteq I$; then for any homogeneous elements $x, y \in B$, $u \in I^{\perp}$, and $v \in I$, we get $b(x u, v)=-(-1)^{\overline{u x}} b(u, x v)=0$ and $b([x, u, y], v)=-(-1)^{\overline{x u}} b([u, x, y], v)=(-1)^{\bar{x}(\bar{u}+\bar{y}+\bar{v})} b(u,[$ $y, v, x])=0$ by $(21)$. Then, $B I^{\perp} \subseteq I^{\perp}$ and $\left[B, I^{\perp}, B\right] \subseteq I^{\perp}$, i.e., $I^{\perp}$ is an ideal of $B$.

We can now prove the following result.

Theorem 2. Let $B=B_{0} \oplus B_{1}$ be a Bol superalgebra such that $\operatorname{str}\left(D_{x, y} L_{z}\right)=0$ for all $x, y, z \in B$. Then, the Killing-Ricci form $\beta$ of $B$ is nondegenerate if and only if the standard enveloping superalgebra $L(B)=B \oplus I P S(B, B)$ is a semisimple Lie superalgebra.

Proof. Let $\alpha$ be the Killing form of the Lie superalgebra $L(B)$. Then, for all $x, y, z \in B, \quad \alpha\left(D_{x, y}, z\right)=\operatorname{str}$ $\left(L_{D_{x, y}} L_{z}\right)=\operatorname{str}\left(D_{x, y} L_{z}\right)$, that is, $\operatorname{str}\left(D_{x, y} L_{z}\right)=0$ if and only if

$$
\alpha\left(D_{x, y}, z\right)=0 \text {. }
$$

Then, by (42) and the invariance of $\alpha$, we have for $x, y, u, v \in B_{0} \cup B_{1}$, 


$$
\begin{aligned}
\alpha\left(D_{x, y}, D_{u, v}\right) & =\alpha([x, y],[u, v]), \\
& =-(-1)^{\overline{x y}} \alpha(y,[x,[u, v]]) \\
& =(-1)^{\bar{x}(\bar{u}+\bar{v}+\bar{y})} \alpha(y,[[u, v], x]) \\
& =(-1)^{\bar{x}(\bar{u}+\bar{v}+\bar{y})} \beta(y,[u, v, x]) .
\end{aligned}
$$

Thus, if $\beta$ is nondegenerate, the restriction of $\alpha$ to $\operatorname{IPS}(B, B) \times \operatorname{IPS}(B, B)$ is nondegenerate, and therefore, $\alpha$ is nondegenerate.

Now by contradiction, suppose that $\beta$ is degenerate. Then, by Lemma $4, B^{\perp}$ is a nonzero ideal of $B$, and therefore, $B^{\perp} \oplus I P S\left(B, B^{\perp}\right)$ is a nonzero ideal of $L(B)$ by Lemma 2 .

By the identities (42) and (43), we obtain

$$
\begin{aligned}
\alpha\left(B^{\perp} \oplus \operatorname{IPS}\left(B, B^{\perp}\right), B \oplus \operatorname{IPS}(B, B)\right)=\alpha\left(B^{\perp}, B\right)+\alpha\left(B^{\perp}, \operatorname{IPS}(B, B)\right) \\
\quad+\alpha\left(\operatorname{IPS}\left(B, B^{\perp}\right), B\right)+\alpha\left(\operatorname{IPS}\left(B, B^{\perp}\right), \operatorname{IPS}(B, B)\right) \\
=\alpha\left(B^{\perp}, B\right)+\alpha\left(B,\left[B^{\perp}, B, B\right]\right)=0 .
\end{aligned}
$$

It follows that $\alpha$ is nondegenerate, and therefore, $L(B)$ is nondegenerate, which ends the theorem.

\section{Data Availability}

No data were used to support this study.

\section{Conflicts of Interest}

The author declares that there are no conflicts of interest.

\section{References}

[1] P. O. Mikheev and L. V. Sabinin, Theory of Smooth Bol Loops in [Russian], UDN, Moscow, Russian, 1985.

[2] L. V. Sabinin, Analytic Quasigroups and Geometry in [Russian], UDN, Moscow, Russian, 1991.

[3] K. Yamaguti, "On Lie triple systems and its generalization," Journal of Science of Hiroshima University, Series A, vol. 21, no. 3, pp. 155-160, 1958.

[4] M. K. Kinyon and A. Weinstein, "Leibniz algebras, Courant algebroids, and multiplications on reductive homogeneous spaces," American Journal of Mathematics, vol. 123, no. 3, pp. 525-550, 2001.

[5] E. N. Kuz'min and O. Zaîdi, "Solvable and semisimple Bol algebras," lgebra and Logic, vol. 32, no. 6, 1993.

[6] V. G. Kac, "Lie superalgebras," Advances in Mathematics, vol. 26, no. 1, pp. 8-96, 1977.

[7] M. Scheunert, The Theory of Lie Superalgebras, Springer, Berlin, Germany, 1979.

[8] M. F. Ouédraogo, "Sur les superalgèbres triples de Lie, thèse de doctorat 3è cycle mathématiques" Ph.D. thesis, Université de Ouagadougou, Ouagadougou, Burkina Faso, 1999.

[9] H. Tilgner, "A graded generalization of Lie triples," Journal of Algebra, vol. 47, no. 1, pp. 190-196, 1977.

[10] S. Okubo, "Parastatistics as Lie-supertriple systems," Journal of Mathematical Physics, vol. 35, no. 6, pp. 2785-2803, 1994.

[11] J. Rukavicka, "Basis of bol superalgebra on one odd generator spanned by words of the length from 1 to 12, workshop 2010," in Proceedings of the 19th Annual CTU Student Scientific Conference, vol. 14, Czech Technical University, Prague, Czech Republic, January 2010.

[12] S. Attan, "A Note on relations between Malcev superalgebras and Bol superalgebras," International Journal of Algebra, vol. 11, no. 11, pp. 181-190, 2017.
[13] T. S. Ravisankar, "Some remarks on Lie triple systems," Kumamoto Journal of Science (Mathematics), vol. 11, pp. 1-8, 1974.

[14] N. Kamiya and S. Okubo, "Quasiclassical Lie superalgebras and Lie supertriple systems," Communications in Algebra, vol. 30, no. 8, pp. 3825-3850, 2002.

[15] J. Peipei and Z. Zhixue, "The Killing forms and decomposition theorems of Lie supertriple systems," Acta Mathematica Scientia. Series B, vol. 29, pp. 360-370, 2009.

[16] P. L. Zoungrana and A. N. Issa, "On killing forms and invariant forms of lie-yamaguti superalgebras," International Journal of Mathematics and Mathematical Sciences, vol. 2017, Article ID 7842482, 9 pages, 2017.

[17] H. Albuquerque, Contribuicôes para a teoria das Superálgebras de Malcev, Universidade de Coimbra, Coimbra, Portugal, 1993.

[18] H. Albuquerque, "Malcev Superalgebras," in Non-associative Algebra and its Applications, S. Gonzalez, Ed., Kluwer Academic Publishers, Dordrecht, Netherlands, 1994.

[19] P. L. Zoungrana and N. B. Pilabre, "On Lie superalgebras involutions and Lie supertriple systems," International Journal of Mathematical Sciences \& Applications, vol. 4, no. 1, pp. 53-71, 2014.

[20] H. Albuquerque and A. Elduque, "A classification of Mal'tsev superalgebras of small dimensions," Algebra and Logic, vol. 35, no. 6, pp. 351-365, 1996.

[21] M. Kikkawa, "On Killing-Ricci forms of Lie triple algebras," Pacific Journal of Mathematics, vol. 96, no. 1, pp. 153-161, 1981. 